\title{
Highlights of Transverse Spin Physics in PHENIX
}

\author{
K. Oleg Eyser \\ Brookhaven National Laboratory \\ Upton, NY 11973, USA \\ E-mail: keyser@bnl.gov
}

Transverse single spin asymmetries are offering unique ways to further our understanding of the dynamic structure of the nucleon. PHENIX has measured $A_{N}$ of different probes at center-of-

mass energies of up to $200 \mathrm{GeV}$ in the past. Asymmetries for $/ / \psi$ mesons have been published previously and we present updated results with increased statistics from the 2012 data set. These asymmetries are expected to be mainly affected by the gluon initial state interactions. The traditional, previous analysis of asymmetries has been compared to a new maximum likelihood method which contains higher moments in the azimuthal distribution. The observed transverse asymmetries are consistent with zero within the statistical uncertainties and in the covered kinematic range.

XXI International Workshop on Deep-Inelastic Scattering and Related Subjects April 22-26, 2013

Marseilles, France 


\section{Introduction}

In a probabilistic way, the nucleon structure is described by parton distribution functions. The unpolarized $q_{i}\left(x, Q^{2}\right)$ and the longitudinally polarized helicity distributions $\Delta q_{i}\left(x, Q^{2}\right)$ are complemented by the transversely polarized distribution functions $\delta q_{i}\left(x, Q^{2}\right)$ (also commonly called transversity). These distributions depend on the partonic momentum fraction $x$ and the momentum transfer $Q^{2}$. Transversity is special in that it is a chiral odd function, i.e., it requires a spin flip in the scattering process in order to be observable. It is, therefore, not accessible in inclusive deep inelastic scattering, and in the hard scattering part of hadron-hadron collisions relies on the exchange of additional gluons in higher-twist effects. As a consequence, transverse single spin asymmetries have been expected to be suppressed at sufficienlty hard scales where perturbative techniques successfully describe quantum-chromodynamics. Surprisingly, very sizable asymmetries $A_{N} \lesssim 40 \%$ have been measured in meson production over a wide range of center-of-mass energies:

$$
A_{N}=\frac{d \sigma^{\uparrow}-d \sigma^{\downarrow}}{d \sigma^{\uparrow}+d \sigma^{\downarrow}}
$$

These asymmetries are largest in the forward direction of the polarized projectile $\left(x_{F} \equiv \frac{2 p_{I}}{\sqrt{s}}>0\right.$, with the longitudinal momentum $\left.p_{l}\right)$ and even persists at energies of up to $\sqrt{s}=500 \mathrm{GeV}$ [1]. Similar asymmetries have also been observed in semi-inclusive deep inelastic scattering over a broad kinematic range.

The apparent contradiction of experimental results with respect to original theoretical expectations has been resolved by the inclusion of initial and final state interactions which are equally important in the process description. These interactions are described in the framework of transverse momentum dependent distributions functions, which goes beyond the onedimensional longitudinal momentum picture. In the initial state, the required chiral-odd part that is needed for a transverse asymmetry can be provided by a correlation of a parton transverse momentum with the nucleon spin (commonly referred to as Sivers effect [2]). The parton itself does not have to be polarized in this case. On the other hand, a spin dependent fragmentation of an outgoing parton can similarly lead to a transverse asymmetry, but only in combination with a non-vanishing transversity distribution (commonly called Collins effect [3]). In a naïve interpretation, the transversity distribution is needed to correlate the fragmentation with the nucleon spin to define (or conserve) the quantization direction. It follows from helicity conservation that the gluon transversity is zero and they do not exhibit Collins asymmetries. From the same argument we can conclude that the transversity of sea quarks is small and transversity should in general be a valence quark effect.

Measurements over a wide range of kinematics are required to disentangle the possible different sources of transverse asymmetries. Also, it is desirable to identify channels that are more exclusively probing the initial or final state effects. Currently, data from proton-proton collisions and from deep inelastic scattering are largely covering different kinematics which has led to questions regarding the universality of the Sivers function. Recent theoretical 
development and a topic of active discussion includes the evolution of transverse momentum dependent distribution functions which will benefit from additional experimental input.

\section{PHENIX at RHIC}

The PHENIX detector has been described in detail elsewhere [4]. The measurements presented here are mainly obtained with the muon systems [5], consisting of three cathode-strip readout tracking chambers within an axial magnetic field. The tracking stations are followed by a muon identifier consisting of Iarocci planar drift tubes and several $10 \mathrm{~cm}$ thick steel absorbers. The relative mass resolution of di-muon pairs is $\frac{\sigma_{M}}{M}=6 \% / \sqrt{M}$ (with $M$ in $\mathrm{GeV}$ ). Signals from the muon identifier can be included in the level-1 trigger selection. The muon detectors cover a pseudorapidity range of $-2.2<\eta<-1.2$ and $1.2<\eta<2.4$.

PHENIX is located in one of the collision points of the Relativistic Heavy Ion Collider, which can collide polarized protons at center-of-mass energies of up to $\sqrt{s}=500 \mathrm{GeV}$. The beam polarizations are measured in separate polarimeters outside of PHENIX [6]. In 2012, transversely polarized protons were used for a recorded integrated luminosity of $L=9.2 \mathrm{pb}^{-1}$ at $\sqrt{s}=200 \mathrm{GeV}$ and average beam polarizations of $P=58 \%$. For single spin asymmetries, we sum over the bunches with alternating polarization directions in one beam, effectively reducing the polarization to zero for this beam. This procedure can be applied to one beam at a time which leads to two uncorrelated measurements of the asymmetries.

\section{Transverse Single Spin Asymmetries of J/Psi Mesons}

Transverse single spin asymmetries have been proposed to be helpful in the understanding of the production mechanism of $I / \psi$ mesons in the presence of a non-vanishing Sivers function of gluons [7]. This transverse asymmetry will be particularly sensitive to the color-singlet channel, whereas contributions from the color-octet cancel between initial and final state effects. Results for $A_{N}(J / \psi)$ have previously been published and have recently been updated after a correction to the analysis based on data from the years 2006 and 2008 [8]. We use the new data from 2012 to confirm that the observed differences between those existing results were indeed statistical fluctuations.

For the current analysis, we employ an unbinned maximum likelihood method to determine the azimuthal, $\varphi$, asymmetries with the following model:

$$
\mathcal{L}=\sum\left(p \cdot a_{1} \cos \varphi+p \cdot a_{2} \cos 2 \varphi+p \cdot a_{3} \cos 3 \varphi\right)
$$

Here, $p= \pm 1$ is the direction of the beam polarization. This model includes higher moments that can arise from higher-twist contributions and others. The unbinned maximum likelihood method is particularly robust with respect to any time dependent variations of the experimental setup. It can, however, be susceptible to cosine-moments in the efficiency in the presence of different luminosities for the two polarization states. Such effects have been carefully studied in toy Monte Carlo simulations in comparison with measured distributions of single muons and di-muons in combination with data from luminosity counters. 
Also, the traditional so-called square-root asymmetry (which is based on integrated yields for left and right detector sides as defined in the polarized scattering frame) is used for consistency checks and to determine systematic uncertainties. It has been shown that differences between the results can be larger than purely statistical uncertainties when the detector acceptance includes areas where the asymmetry is suppressed by the geometry of the polarized collision. This effect is in fact driven by statistical fluctuations in an acceptance range where the asymmetry is close to zero. The additional uncertainty can be as large as half of the statistical uncertainty; it is independent of the probe or the dataset and is intrinsic to the azimuthal modulation of the asymmetry. A new figure-of-merit has been determined from toy Monte Carlo studies, which leads to a reduced acceptance $\Delta \varphi / \pi=\operatorname{acos}(0.5)$. For this new figure-ofmerit, the systematic uncertainty is estimated to be less than 0.2 of the statistical uncertainty.

The transverse single spin asymmetry $A_{N}$ in the above model is folded with the beam polarization $P$ into the parameter $a_{1}$. The asymmetries are determined for di-muon pairs of opposite charge in the $I / \psi$ mass range $\left(A_{N, \text { incl }}\right)$ and in a background region $\left(A_{N, b g}\right.$, at low masses). The physics observable $A_{N}$ is then corrected for an asymmetry of the background fraction $r$ :

$$
A_{N}=\frac{A_{N, i n a l}-r A_{N, b g}}{1-r}
$$

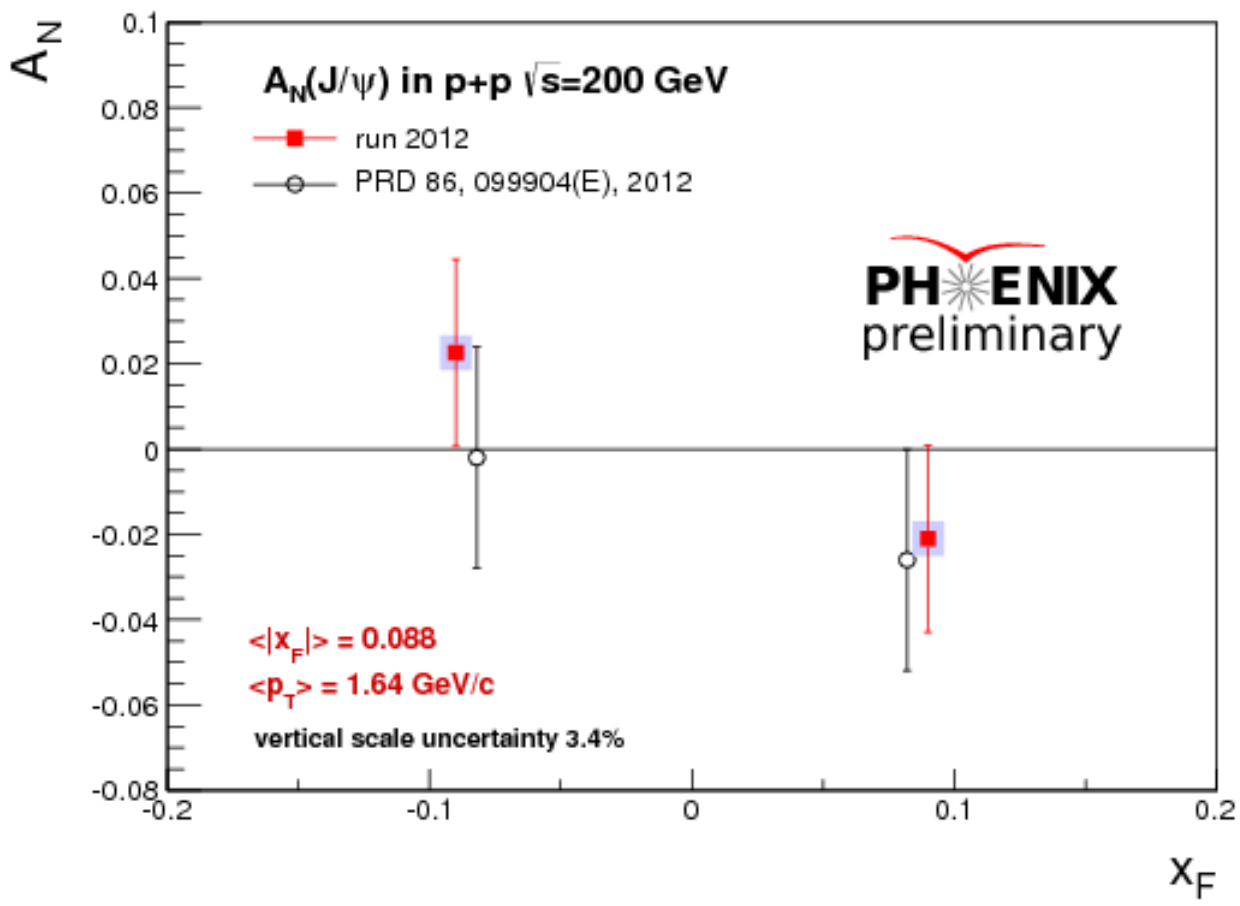

Figure 1:Transverse single spin asymmetries $A N$ of $W \psi$ mesons as function of $x_{F}$. Uncertainties are statistical (bars) and systematic(boxes). An additional global scale uncertainty of $3.4 \%$ is coming from the determination of the beam polarizations. 
Figure 1 presents the final asymmetries with statistical and systematic uncertainties and compares the results with [8] as function of $x_{F}$. The results are well consistent within statistical uncertainties and increase our confidence that the observed fluctuations between the previous subsets of data were indeed statistical. The asymmetries are also consistent with zero in the covered kinematic range. The other azimuthal, higher moments, $a_{2}$ and $a_{3}$, have been found to be consistent with zero.

\section{Conclusion and Outlook}

PHENIX has measured transverse single spin asymmetries $A_{N}$ of various probes at different center-of-mass energies. We presented new results of $I / \psi$ mesons at $\sqrt{s}=200 \mathrm{GeV}$ from the new 2012 data with increased statistics compared to previous measurements. The observed asymmetries are consistent with zero within the covered kinematic range. This is similar to other probes with comparable kinematics. Nonvanishing asymmetries are typically only starting to appear in more forward directions $x_{F}>0.2$ and a very weak dependence on the center-of-mass energy.

Transverse asymmetries in high energy hadronic collisions are a theoretically challenging topic, which helps to improve our understanding of the dynamic structure of the nucleon and the process dependence of color interactions. In order to disentangle different effects in the initial and final state interactions, it is essential to provide measurements over a wide kinematic range and to identify and enable probes that are more exclusive with regards to certain effects. Obvious candidates are Drell-Yan production and direct photons, which are in the focus of near and mid-term detector upgrades.

\section{References}

[1] S. Heppelmann, these proceedings

[2] D.W. Sivers, Phys. Rev. D41, 83 (1990)

[3] J.C. Collins, Nucl. Phys. B396, 161 (1993)

[4] M Harrison, T. Ludlam, S. Ozaki (editors), NIM A499, issues 2-3, 235-880 (2003)

[5] H. Akikawa et al., NIM A499, 537-548 (2003)

[6] https://wiki.bnl.gov/rhicspin/upload/6/68/Run91112_results.pdf

[7] F. Yuan, Phys. Rev. D78, 014024 (2008)

[8] A. Adare et al., Phys. Rev. D82, 099904(E) (2012) 